\title{
52-13 Dispersal of Volcanic Ash on Mars: Ash Particle Shape Analysis
}

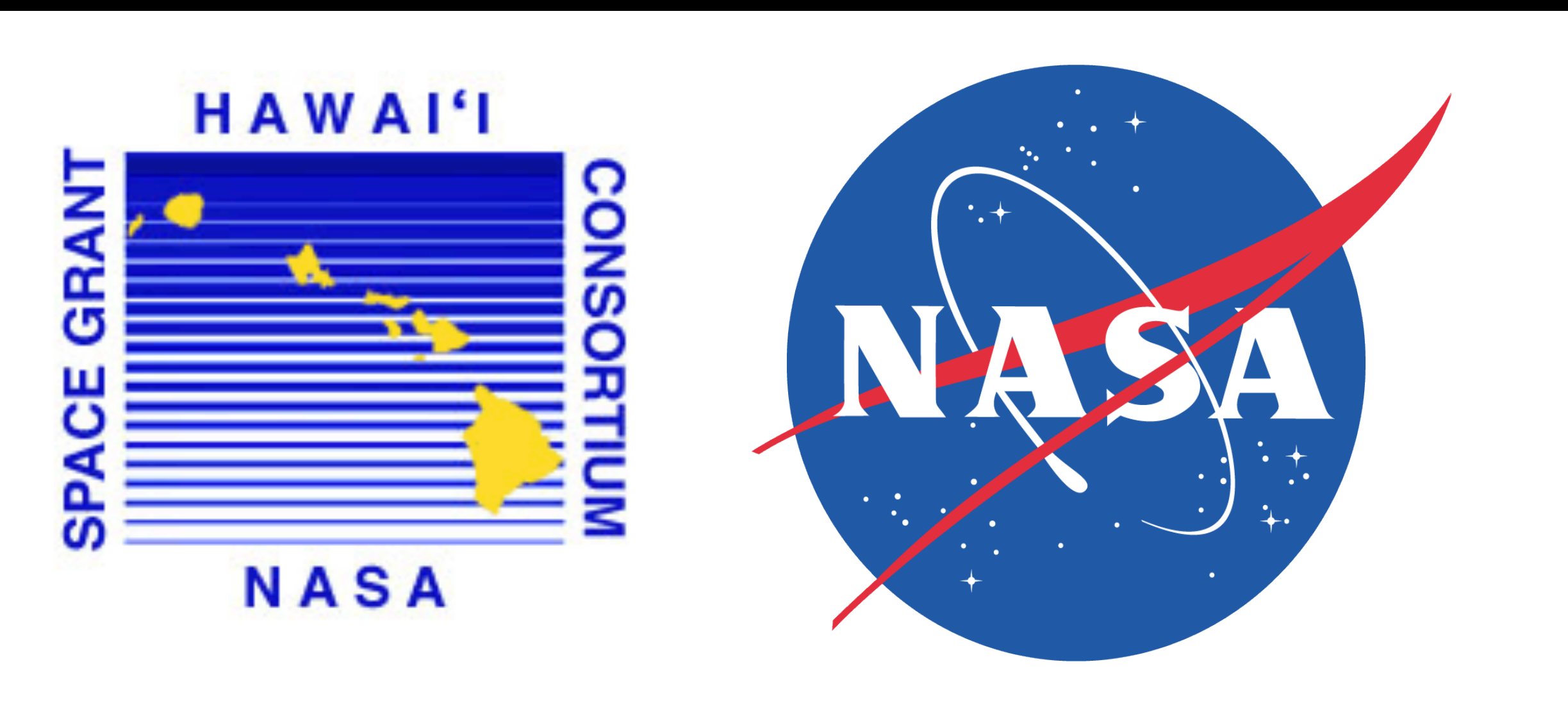

\section{Project Goals}

Determine whether certain fine-grained Martian deposits are volcanic in origin by using mathematical dispersal modeling.

\section{Improvements on Previous Work}

Current settling models use spheres as ash analogs in drag calculations. These simplifications introduce inaccuracies in the treatment of drag coefficients leading to inaccurate dispersal predictions.

\section{Hypothesis}

Through incorporating shape parameters of volcanic ash particles into mathematical dispersal models we will be able to improve the accuracy of dispersal distance estimates. Since natural ash particles tumble, drag forces act on a changing cross sectional area. Therefore, capturing images of each grain in three unique orientations ensures that we capture a more robust set of shape parameters for each grain.

\section{Methods}

We selected five, 1-2 mm volcanic ash grains that display the full range of morphologies present in basaltic volcanic ash, including blocky, equant grains, elongate grains, and grains with a highly irregular surface morphology.

Silhouette images of the grains, imaged using a Nikon SMZ 1500, are captured at a resolution of 375 pixels $/ \mathrm{mm}$. These images are converted to a binary (black/white) form using Photoshop. The shape

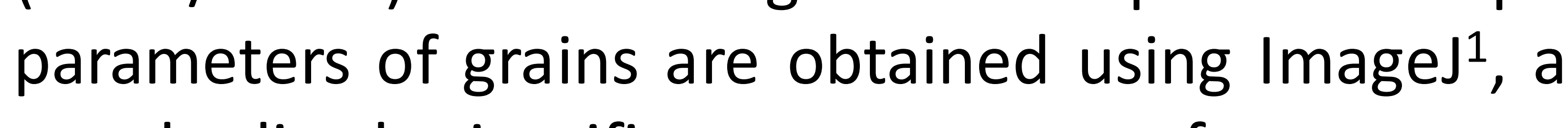
standardized scientific measurement software.

\section{Shape Parameters}

Shape parameters are unitless mathematical values that quantitatively describe geometric features. They can be used to inform the choice of drag coefficients in dispersal models. They are gathered in an automated replicable process using ImageJ. We focused on two main parameters: aspect ratio $(A R)$, which describes the degree of elongation of the grain, and convexity, which describes the degree of surface irregularity. A sphere would produce a value of $\mathbf{1}$ for both aspect ratio and convexity.
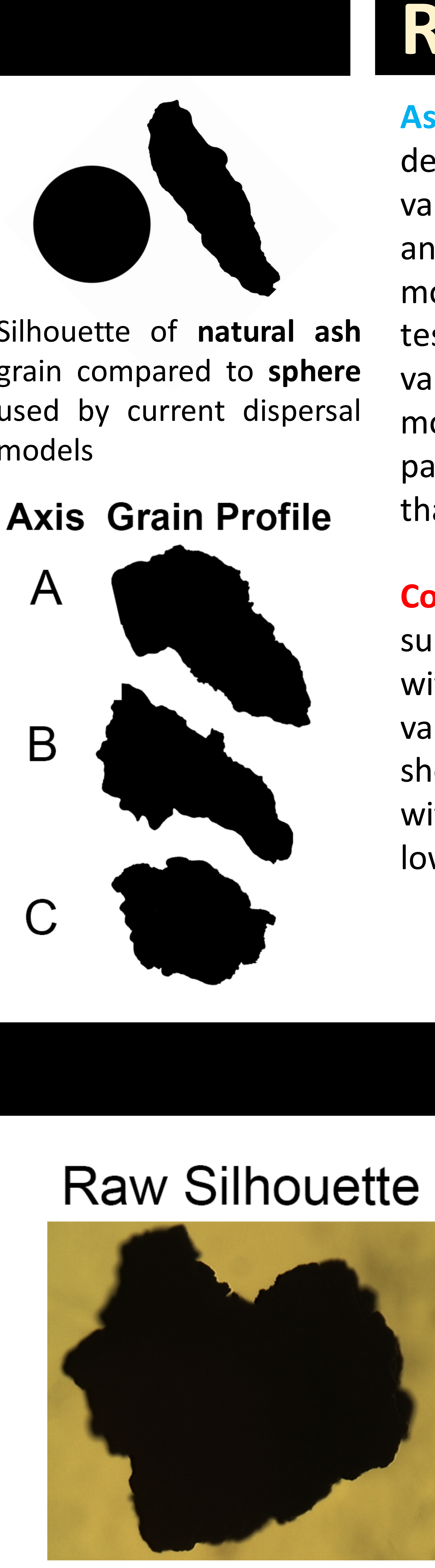

Binary

quantifies the surface irregularities of a grain with a perfect sphere having a value of 1 . Natural grains showed values lower than 1 with the highest being 0.9 and lowest being 0.83 .

ReSUltS
Aspect ratio quantifies the
degree of elongation, with
values close to 1 being equant
and values larger than 1 being
more elongated. Grains in our
test set varied from 1.2 to $\mathbf{3 . 3}$,
values that provide a much
more realistic range of
parameters compared with
that of a sphere (AR = 1 ).
Convexity quantifies the
surface irregularities of a grain,
with a perfect sphere having a
value of 1. Natural grains
showed values lower than 1
with the highest being 0.9 and
lowest being 0.83 .

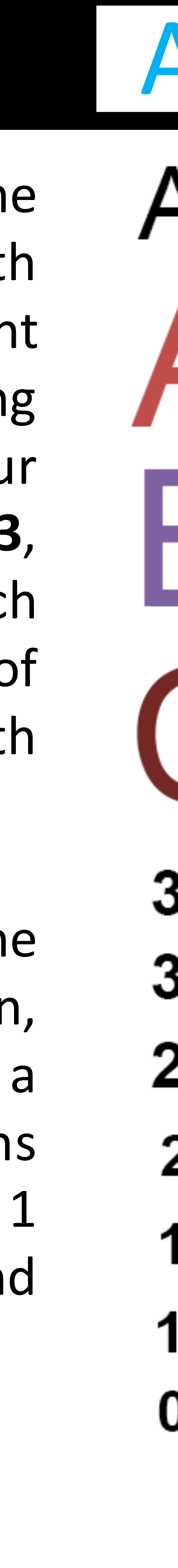

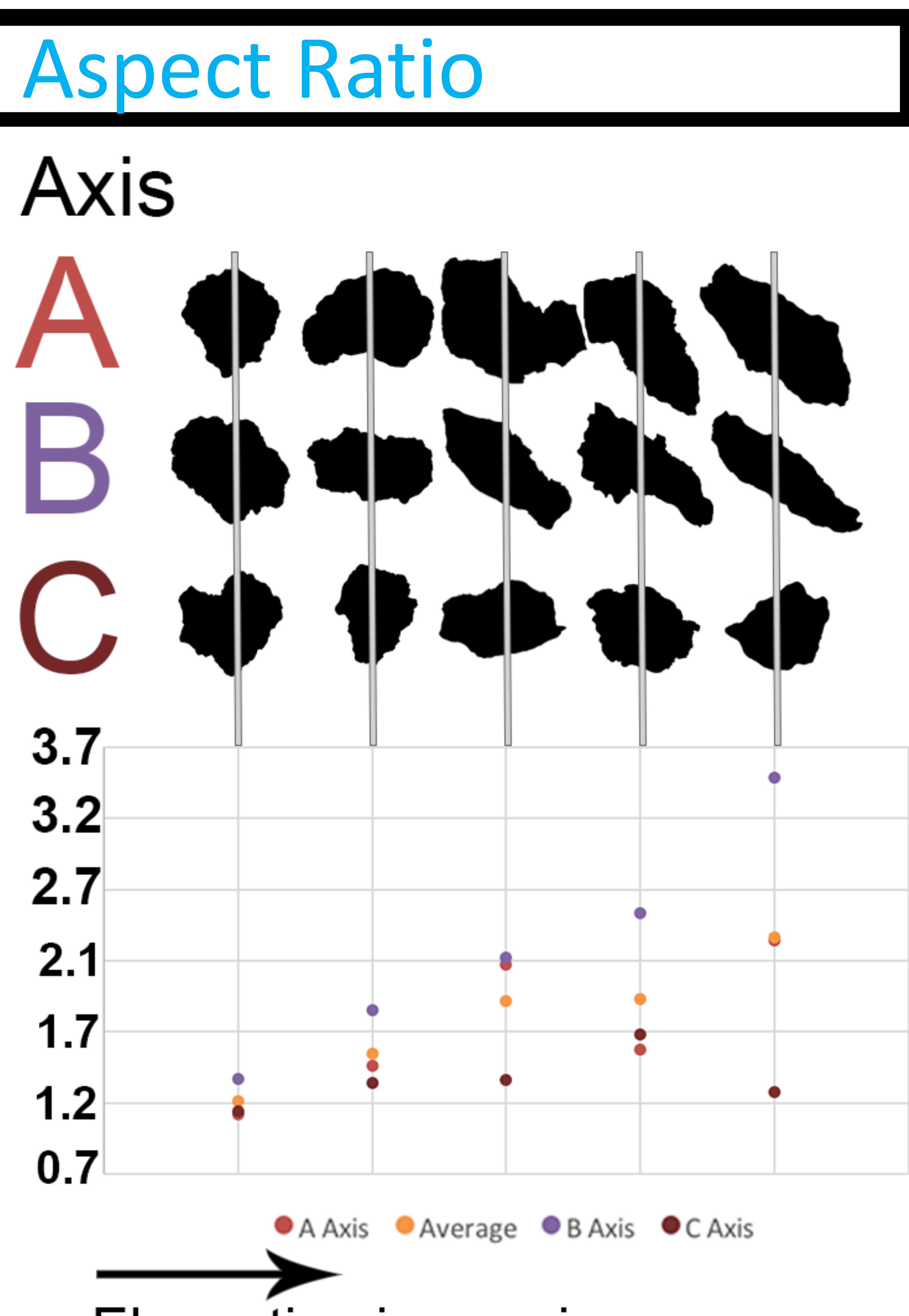

Elongation increasing

\section{HIGP}

Geology

Cueophysics

\section{Future Work}

By incorporating shape parameter data from natural ash grains, the accuracy of our dispersal models will be greatly improved. We plan to extend this analysis to samples from additional eruptions exhibiting a range of intensities and particle sizes that would be expected for Martian volcanism.

This future work involves expanding analysis to

\section{Convexity}

Axis
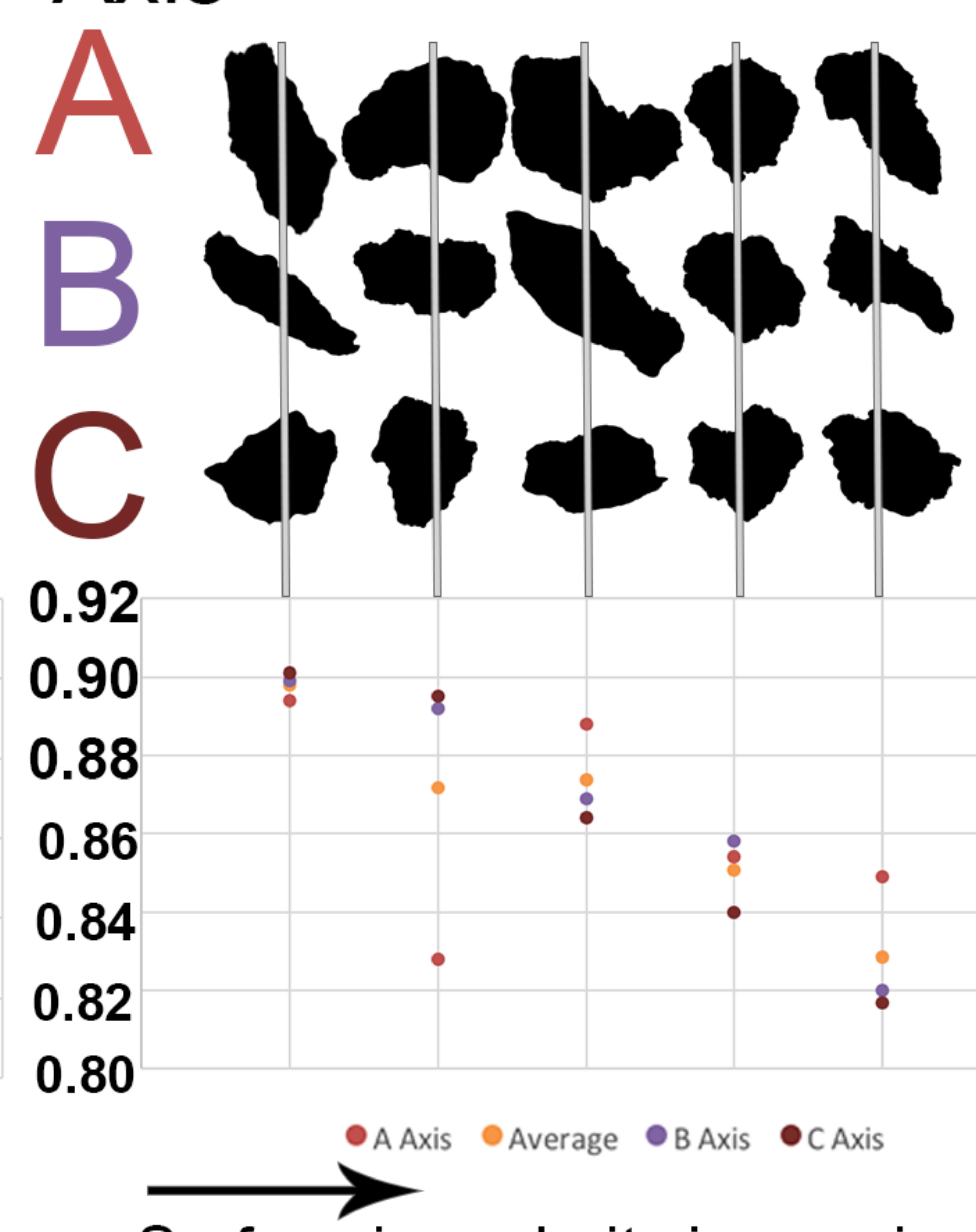

Surface irregularity increasing

30 grains in each of 6 size fractions between 1 $\mathrm{mm}$ and $64 \mu \mathrm{m}$ for each eruption. This will enable us to create a database of statistically robust shape parameter data for use in choosing appropriate drag coefficients for dispersal modeling.

We look to image these additional grains with a focus stacking method, where multiple images are taken at various depths of field and merged together to create a completely in-focus image. This will allow us to capture fine grain detail on ash particles that will provide even better data. Convexity, being highly dependent on surface irregularity, should be most improved by focus stacking.

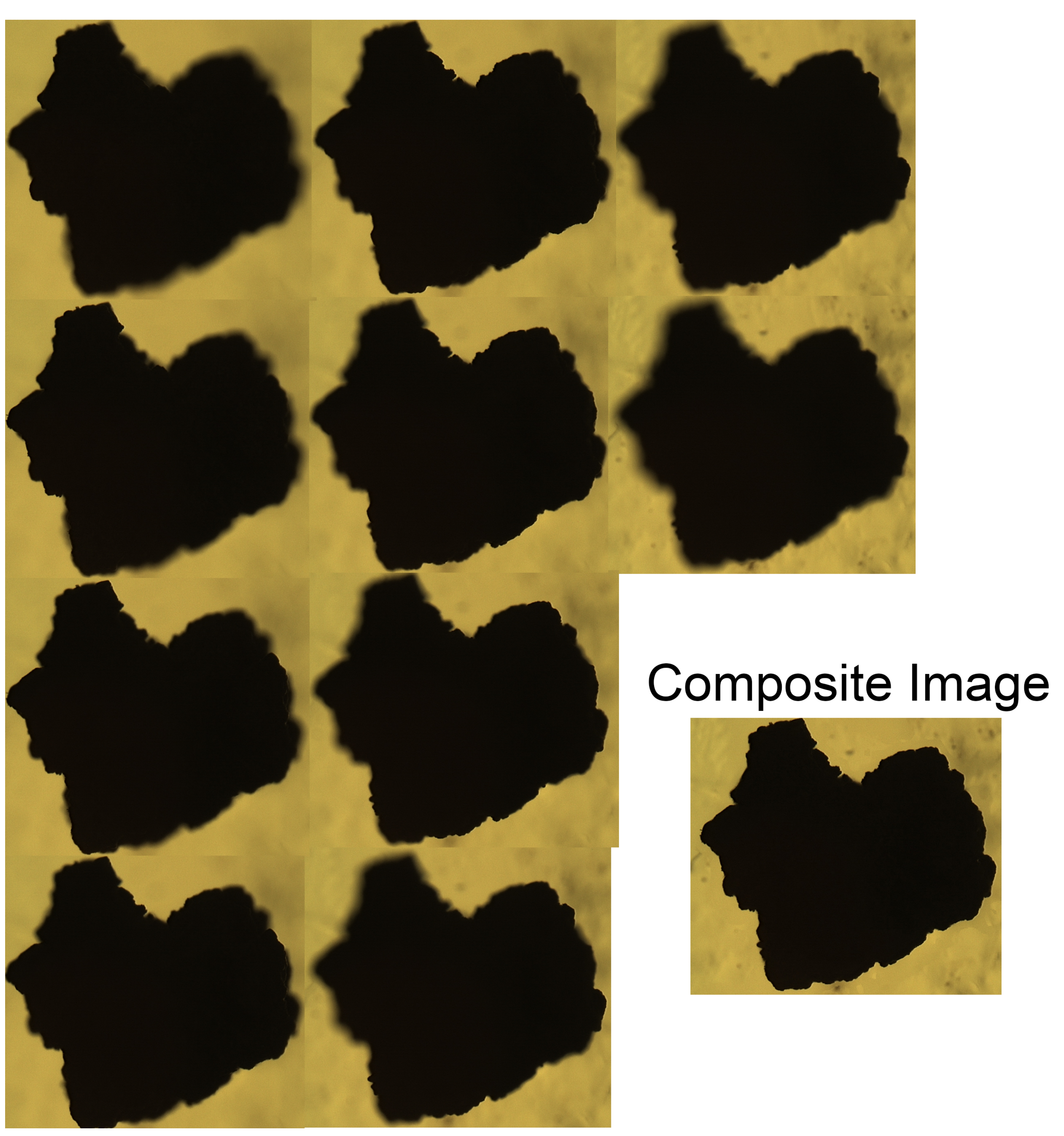

\section{Acknowledgments and References}

This project would not have been possible without mentorin by Dr. Sarah Fagents and Erin Fitch. We would like to thank Dr. Gary Huss for generous access to his facilities for image acquisition.

${ }^{1}$ Schneider CA, Rasband WS and Eliceiri KW 2012. NIH Image to ImageJ: 25 years of image analysis. Nature Methods, pp. 671, doi:10.1038/nmeth.2089 